\title{
Germanica
}

\section{Gottfried Benns autobiographische Schriften als Reaktionen auf die kollektive germanische Dummheit}

Les récits autobiographiques de G. Benn

\section{Rammon Reimann}

\section{CpenEdition}

Journals

Édition électronique

URL : http://journals.openedition.org/germanica/2051

DOI : 10.4000/germanica. 2051

ISSN : 2107-0784

Éditeur

Université de Lille

\section{Édition imprimée}

Date de publication : 30 juin 1997

Pagination : 125-136

ISBN : 9782098426320

ISSN : 0984-2632

Référence électronique

Rammon Reimann, «Gottfried Benns autobiographische Schriften als Reaktionen auf die kollektive germanische Dummheit », Germanica [Online], 20 | 1997, Online erschienen am: 16 Juli 2013, abgerufen am 06 Oktober 2020. URL : http://journals.openedition.org/germanica/2051 ; DOI : https:// doi.org/10.4000/germanica.2051

Ce document a été généré automatiquement le 6 octobre 2020.

(c) Tous droits réservés 


\section{Gottfried Benns autobiographische Schriften als Reaktionen auf die kollektive germanische Dummheit}

Les récits autobiographiques de G. Benn

Rammon Reimann

Wer das Hohe an einem Menschen nicht sehen

will, blickt um so schärfer nach dem, was niedrig und Vordergrund an ihm ist - und verräth sich selbst damit. Nietzsche, Jenseits von Gut und Böse, Aph. 275.

Die Verdummung in D'land ist unglaublich. E.-R. Curtius an Benn, 27. Mai 1949.

1 Gottfried Benns erste autobiographische Schrift fällt sehr kurz aus: «Geboren 1886 und aufgewachsen in Dörfern der Provinz Brandenburg. Belangloser Entwicklungsgang, belangloses Dasein als Arzt in Berlin.» Sie stammt aus dem Jahre 1919 und wurde abgefaßt für die von Kurt Pinthus herausgegebene Lyriksammlung Menschheitsdämmerung ${ }^{1}$.

sagt ein Dichter: Wenn Sie etwas über mich erfahren wollen, dann lesen Sie meine Gedichte. Benedetto Croce, der zu umecht in Vergessenheit geraten ist, schlug sich seinerzeit auf die Seite der Dichter, als er bemerkte: «Der Dichter ist also nichts anderes als seine Dichtung: diese Feststellung ist nicht paradox, wenn man bedenkt, daß der Philosoph nichts anderes als seine Philosophie und der Staatsmann nichts anderes als seine Tat und sein politisches Werk ist» ${ }^{2}$.

Dichtung ist für Benn wie für jeden Dichter der Versuch, individuelles Unbehagen an der Kultur durch das Wahre zu lindern. Um Geringeres als um das Wahre ging es Benn nicht, und weil er Nietzsche gelesen hatte, mit dessen Ästhetik er sich weitestgehend 
identifizierte ${ }^{3}$, wußte er, daß das Wahre nur durch das Schöne transparent wird, selbst wenn dieses Schöne das Häßliche ist, ohne das es nicht existierte.

Es ist nicht irgendein Dichter, der hier ausschließlich auf seine Dichtung verweist; Gottfried Benn war der letzte Dichter deutscher Sprache, ganz im Sinne von Croce, der, obwohl er mit der aristotelischen und der auf ihr fußenden Gattungstheorie der Anciens wenig am Hut hat, Dichtung und Literatur scharf trennt ${ }^{4}$. Wohl kamen nach Benn noch deutsche Gedichte, deutscher Dichter indes kam keiner mehr in Sicht. Fast jedes Gedicht Gottfried Benns ist selbständige Existenz.

1928 erscheint Epilog und lyrisches Ich ${ }^{5}$ als Essay in der Gesammelten Prosa. Dort ist zu lesen: «... siebenunddreißig Jahre und total erledigt, ich schreibe nichts mehr.» ${ }^{6}$ Natürlich alles ironisch. Dr. med. Gottfried Benn schrieb weiter, weil Dichtung und nicht die Behandlung von Trippern sein Fernbestimmtes war. Über die Zunft der Mediziner und das medizinische Handwerk hatte er sich schon 1920 vor Medizinstudenten geäußert: «... ich will Mißtrauen säen in Ihre Herzen gegen Ihrer Lehrer Wort und Werk, Verachtung gegen das Geschwätz vollbärtiger Fünfziger, deren Wort der Staat lohnt und schützt, und Ekel vor einem Handwerk, das nie an eine Schöpfung glaubte.» ${ }^{7}$ Dies korrespondiert mit Nietzsches Schöpfungsbegriff: «Im Verhältnisse zu einem Genie, das heisst zu einem Wesen, welches entweder zeugt oder g ebiert, beide Worte in ihrem höchsten Umfange genommen -, hat der Gelehrte, der wissenschaftliche Durchschnittsmensch immer etwas von der alten Jungfer: denn er versteht sich gleich dieser nicht auf die zwei werthvollsten Verrichtungen des Menschen.» ${ }^{8}$

Das Berlin der Zwanzigerjahre, wo Benn als Arzt für Hautund Geschlechtskrankheiten praktizierte, ist der Sündenfall weil die amerikanischste Stadt Europas, das sich von Paris aus mit dem Surréalisme ${ }^{9}$ noch einmal verzweifelt zu verteidigen versucht hatte gegen die Übermacht des Unausweichlichen. Wenige sahen das Menetekel, wenige durchlitten das. Benn war einer der Wenigen, während die Berliner Durchschnittsfamilie sich über Schilder in Gartenkneipen freute, auf denen verzeichnet war: «Hier dürfen Familien Kaffee kochen»; das war Freiheit.

7 Freilich sind die Menetekel der Zeit, ist das Leiden an der Gegenwart immer nur Metapher dafür, daß das Leben generell und zu allen Zeiten abzuleiden ist ${ }^{10}$. Geeigneter als Berlin ist kein Ort der Welt zu dieser Zeit, um des zu überwindenden Zeitgeists innezuwerden. Der Amerikanismus ${ }^{11}$ trifft hier in der Metropole auf den deutschen Frust nach dem Friedensvertrag von Versailles, der «die epochemachende diplomatische Fehlleistung dieses Jahrhunderts darstellt» ${ }^{12}$. Der deutsche Masochismus, der sich von der Gründerzeit bis zum Ersten Weltkrieg in Größenwahn verkehrt, tobt sich während der Zwanzigerjahre, vor allem in Berlin, als schier ungebremster Hedonismus aus, bevor er unter den Nazis durch einen gesamtgesellschaftlichen Konsens des Sadismus kompensiert wird ${ }^{13}$ Nosce te ipsum ist der deutschen Seele spätestens seit 1871 und bis auf den heutigen Tag fremd. Ausnahmen bestätigen hier nur die Regel und dürfen mit Nachsicht oder gar Verständnis nicht rechnen. Und gerade durch den Umstand, dass Deutschland seine hervorragendsten Geister: Heine, Wagner, Marx, Engels, Nietzsche, Thomas Mann, Benjamin, Adorno, Einstein, um nur einige zu nennen, von jeher ins Exil getrieben, zum Schweigenoder kurzerhand umgebracht hat, ist es zum Verharren in selbstgefälliger Borniertheit gezwungen. 
8 Manch Deutscher, der der Weimarer Republik ausgesetzt war, irgend fühlen konnte, und dessen IQ nicht im Minusbereich angesiedelt war, mußte dies Staatsgebilde zum Erbrechen finden. Gottfried Benn war weder geistig minderbedarft noch unsensibel. Dergleichen ist tragisch - «dumm sein und Arbeit haben: Das ist Glück.» ${ }^{14}$

9 Summa summarum erscheint 1926 in Ossietzkys Weltbühne. Auch das noch Spiel, noch nicht Wirklichkeit ${ }^{15}$. In Summa summarum geht es ums Geld, von dem seit Marx und Freud jeder weiß, was es ist. Der deutsche Dichter Gottfried Benn rechnet der Welt vor, was Dichtung wert ist: Vier Mark fünfzig im Monat. Dabei hatte ihn kein Geringerer als «Herr Soupault zu den fünf größten Lyrikern nicht nur Deutschlands, sondern Europas» gezählt ${ }^{16}$.

«Willkommen in Sils-Maria». Das steht als Motto unter dem Titel dieses autobiographischen Lebensrückblicks von 1926 und spielt auf Nietzsches materielle Not $\mathrm{an}^{17}$. Für Genie gibt es selten Geld, Mediokrität ist lukrativ. Benn wußte das, und er litt darunter, obwohl er das wußte. «Aber, wie gesagt, ich beklage diesen Zustand nicht», heißt es in Summa summarum. «Beklagte ich ihn, müßte ich die Gesellschaftsordnung beschuldigen, aber die Gesellschaftsordnung ist gut. [...] Diese Politiker und Minister, was verjauchen sie nicht alles rhetorisch vom Pfingstwunder bis zur Apokalypse [...]..»18 Resignativ schließt Summa summarum: «... - alles für vier Mark fünfzig pro Monat, aber ich zurück zu meinen Trippern und jeden Monat ein Gedicht! Gedicht ist eine unbesoldete Arbeit des Geistes, der Fonds perdu, eine Art Aktion am Sandsack: einseitig, ergebnislos und ohne Partner - : evoë!» ${ }^{19}$

11 Dem ging die Kunst nicht nach Brot, und insofern war er ein Fossil. Die Zeitung, die im letzten Drittel des 19. Jahrhunderts die Menschheit profund zu verblöden sich anschickte $^{20}$, hatte das Phänomen des Literaten gezeitigt, der für Geld schrieb und davon auch leben konnte, mehr oder weniger. Der Dichter Gottfried Benn verweigerte sich den Gesetzen des Marktes ${ }^{21}$, er hatte, als Dichter, den Markt nicht nötig. Kunst als Ware mit Tauschwert ist korrumpierte Kunst.

Spätestens vom 27. Februar 1933 an war in Deutschland nichts mehr, wie es vorher war. Der Führer Adolf, dem die Weimarer Republik nach dem 9. November 1923 einen Sanatoriumsaufenthalt mit Sekretär bewilligt hatte, setzte sein bei dieser Gelegenheit diktiertes literarisch-philosophisches Hauptwerk nach dem Reichstagsbrand offen und terroristisch in die Tat um, und er hatte dabei tatkräftige Helfer. In allen Bevölkerungsschichten.

Gottfried Benn hat Mein Kampf nie gelesen; er ist überhaupt nicht auf die Idee gekommen, dieses Buch anzufassen, und das war ein Fehler. Es hätte ihm einen Irrtum erspart. Benns politisches Desinteresse führt im Verbünde mit dem nur allzu begründeten Ressentiment gegen die Weimarer Republik kurzzeitig dazu, daß er glaubt, von den neuen Machthabern, die er zu dieser Zeit allerdings als Verbrecher noch nicht erkannt hat, durch scheinbares Entgegenkommen in Ruhe gelassen zu werden. Dokumentiert ist dies u. a. in der Rundfunkrede Der neue Staat und die Intellektuellen und in der Antwort an die literarischen Emigranten von 1933. Dieser Irrtum geht freilich auch mit dem Umstand einher, daß Benn sich existentiell bedroht fühlt von den neuen Machthabern, die weitaus weniger Spaß verstanden als die Weimarer Jauchepolitiker. Bereits am 22. April 1933 hatte Oskar Loerke in sein Tagebuch notiert: «Benn meinte, wir würden nicht nur ausgeschaltet, sondern auch körperlich vernichtet werden. $»^{22}$ Angesichts solch realer und auch als real empfundener Bedrohung ist die Frage zu stellen, wie Benn auf den Brief von Klaus Mann hätte reagieren sollen? 

verließen, aber er taugte nicht für das Exil, auch nicht für den organisierten Widerstand. «Ich persönlich hatte keine Veranlassung, Berlin zu verlassen, ich lebte von meiner ärztlichen Praxis und hatte mit politischen Dingen nichts zu tun» ${ }^{23}$, schreibt er dazu in Doppelleben. Wäre Gottfried Benn im Exil draufgegangen oder in einem Konzentrationslager, dann hätten einige gute Menschen ihn als guten Menschen betrauern können, und es ist gut für die guten Menschen, daß sie immer wissen, was gut und was böse ist. Gottfried Benn war kein guter Mensch; auch seine Dichtung steht jenseits von Gut und Böse.

Die Wunde Weimar sitzt tief, und Benn versucht, die Zeichen der neuen Zeit zu verdrängen. Am 21. Oktober 1933 heißt es in einem Brief an Gertrud Hindemith: «Wenn jetzt die Abgetakelten schrein: was wird aus der Kunst, denke ich bei mir: die Epigonen des II. Reichs sind nicht schützund pflegebedürftiger als die Dilettanten im III. Wer damit nicht fertig wird, soll die Schn[auze] halten, wer was ist, wird damit fertig. Hier ist Stoff $u$. inneres Erlebnis - ran! Hier ist Geschichte - ertrage sie. Hier ist Schicksal friß Vogel oder stirb! Gefahren, Untergang - liebe sie! Amor fati» ${ }^{24}$.

niemand kann in seiner Zeit leben und zugleich frei von ihr sein. Spätestens seit Ende der Zwanzigerjahre stand Benn im Kreuzfeuer der ideologischen Auseinandersetzungen ${ }^{25}$. Er war jedoch alles andere als ein homo politicus, forderte immer wieder für den Dichter die Freiheit, sich gegen den Zeitgeist abzuschließen ${ }^{26}$ und stellte selbst im Vorwort zu Der neue Staat und die Intellektuellen klar: «Das Schöpferische ist weder rechts noch links, sondern immer zentral.» ${ }^{27}$

Gottfried Benn hatte weder bei linken noch bei rechten Eiferern je Freunde. Bereits 1933 wird seine Lage insofern existentiell bedrohlich, als der nationalsozialistische Ärztebund ihn von einer Liste strich, auf der Ärzte standen, die bestimmte Atteste ausstellen durften, und auch die Angriffe von im III. Reich als Schriftsteller geltenden Dummköpfen ließen nicht lange auf sich warten. Im April 1934 denunziert der Balladenbarde Börries von Münchhausen Benn in einem an Hinterhältigkeit nicht zu übertreffenden Brief als Juden ${ }^{28}$, und dies, nachdem Goebbels anläßlich der Bücherverbrennung am 10. Mai 1933 unmißverständlich verkündet hatte: «Das Zeitalter eines überspitzten jüdischen Intellektualismus ist nun zu Ende» ${ }^{29}$.

Mit Ahnenschwierigkeiten, einem autobiographisch-genealogischen Abriß, der wenig später in Lebensweg eines Intellektualisten unter der Kapitelüberschrift Die Erbmasse nachgedruckt wird, verteidigt Benn, der seines expressionistischen Frühwerks wegen ohnehin als entartet galt, seine Haut. Ohne Zweifel ist diese unter einer Zwangssituation und sehr rasch entstandene Schrift peinlich; weil die Adressaten peinlich sind, die sie herausforderten. Dennoch: Die Kunst und damit sich selbst hat Benn in Lebensweg eines Intellektualisten nicht verraten. Er unterscheidet hier, nicht ohne vorher seine romanische Abstammung hervorgehoben $\mathrm{zu}$ haben, zwischen Kunstträger und Kulturträger, ähnlich wie der von ihm zeitlebens verehrte Thomas Mann 1919 in den Betrachtungen eines Unpolitischen Kultur und Zivilisation unterschied, um damit die Franzosen abzukanzeln. Benns ästhetischer Diskurs ähnelt dem Thomas Manns, kehrt dessen chauvinistische Ausfälle jedoch um. Als Kunstträger sieht Benn Baudelaire, Flaubert, Nietzsche, George, in deren Tradition er sich begreift, als Kulturträger all jene seit Piaton, die gesellschaftliche Intentionen haben, am Ephemeren hängen, denen Wahrheit nicht zugänglich ist. Dies bedeutet nichts anderes als Verachtung der Macht und Geringschätzung des Staates, gerade durch den Verweis 
auf Piaton ${ }^{30}$. Die großen Geister Frankreichs und Deutschlands sind für Benn eines: Träger der Kultur, "statistisch asozial»" ${ }^{31}$ und der Rest ist Schweigen. Oder die Menschheit ${ }^{32}$.

Benns Argumentation geht, insofern sie Kunst über das Ephemere stellt, auch über die des von ihm bewunderten George hinaus, der gesagt hatte: «Die Wahrheit ist in jedem Äon eine andere», worauf der sich naiv gebende Ernst Robert Curtius zurückfragte: «Es muß aber doch ein Letztes über den wechselnden Äonen geben?» George fährt daraufhin jäh auf und herrscht Curtius an: «Ja, das gibt es. Aber das geht Sie nichts an. Mit dem darf selbst ich mich nicht befassen.» ${ }^{33}$

Nach Lebensweg eines Intellektualisten folgt die große Autobiographie Doppelleben (1950), die Benn wohl ohne das Drängen seines Verlegers Max Niedermayer nie geschrieben hätte. Also liegt dieser Autobiographie, wie der vorangegangenen, eine Nötigung zugrunde, wenn auch diesmal eine freundliche. Es gehört zur deutschen Paranoia und ist, nebenbei, ein Signum des provinziellen wie des totalitären Staats, daß Dichter und Schriftsteller dortzulande bis auf den heutigen $\mathrm{Tag}^{34}$ genötigt werden, der kollektiven germanischen Dummheit Erklärungen über ihr Werk hinaus zu geben. Benn wußte indes sehr genau, was er von der Öffentlichkeit und von Politik zu halten hatte, wie aus einem Brief an Thilo Koch vom 12. Oktober 1950 hervorgeht: «Die Öffentlichkeit ist der Gestank einer Senkgrube und die Politik ist das Gebiet von Reduzierten.» ${ }^{35}$

Über Doppelleben ist viel geschrieben worden und über Benns Irrtum hinsichtlich des Nationalsozialismus auch. Das alles hier aufzulisten und womöglich noch $\mathrm{zu}$ kommentieren geriete ins Uferlose und ist auch der Mühe nicht wert. Jürgen Schröders Feststellung trifft den Nagel auf den Kopf: «Benn hat lange genug als Medium der Selbstbefragung und Vergangenheitsbewältigung der Deutschen gedient, er hat oft genug als Sündenbock und Ersatzopfer im apologetischen Selbstgericht der Deutschen herhalten müssen.» ${ }^{36}$ Dabei befindet sich Benn nicht in der schlechtesten Gesellschaft; Hölderlin gehört dazu, Nietzsche, George, Spengler, Heidegger, Jünger und neuerdings wieder Ernst Robert Curtius ${ }^{37}$. In diesen Schlammschlachten wird versucht, ob bewußt oder unbewußt ist hierbei ohne Belang, an herausragenden Geistern die in Deutschland nie bewältigte Nazivergangenheit endlich in den Griff zu kriegen. Auch dies ist ein Beitrag zur deutschen Paranoia. Begleitet ist dieser Umgang mit Benn von unfreiwilliger Komik, wovon die Wissenschaft, wie spätestens seit Flauberts Dictionnaire des idées reçues bekannt ist, immer genügend parat hat: «Benn selbst hat es oft ausgesprochen: der moderne Dichter reicht mit den Antennen seines Wesens in das zeitgenössische Geschehen hinein. Es ist sein hervorstechendes Charakteristikum, daß er reflektiert - ganz allgemein über Strukturen und Vorkommnisse seiner Zeit und

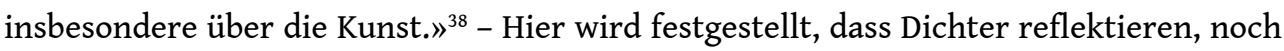
dazu über Kunst - ungeheuerlich; und hier wird gelogen, denn von «den Antennen seines Wesens» hat Benn nie etwas verlauten lassen. Gottfried Benn fühlte sich nicht in der Nachfolge Friederike Kempners, eher in der Nietzsches, obgleich Else Buddeberg von "seiner nicht sehr gründlichen Nietzsche-Aneignung»" genauestens unterrichtet zu sein scheint und diesen Mangel an deutscher Gründlichkeit als eine Ursache für Benns Irrtum 1933 ansieht ${ }^{40}$.

Diesen Irrtum sah Benn rasch ein, schon 1934, nach der sogenannten Röhm-Revolte, und er bekennt diesen Irrtum auch in Doppelleben als solchen: «Dieser Siebenundzwanzigj ährige», heißt es mit Bezug auf Klaus Mann, «hatte die Situation richtiger beurteilt, die Entwicklung der Dinge genau vorausgesehen, er war 
klarerdenkend als ich, meine Antwort [... ] war demgegenüber romantisch, überschwenglich, pathetisch $»^{41}$.

Klaus Manns Achtung vor Benn blieb, und in dieser geistigen Noblesse unterscheidet sich Klaus Mann von seinen pathischen Volksgenossen der Nazizeit und des Nachkriegs: In Doppelleben äußert sich Benn kurz zu Klaus Manns Roman Mephisto, den Klaus Mann ihm «1937 mit einer reizenden und melancholischen Widmung schickte», und der Benn auch erreichte ${ }^{42}$.

Benn wählte 1935, nachdem er seinen Irrtum eingesehen hatte, den Eintritt als Arzt in die Armee als «aristokratische Form der Emigration» ${ }^{43}$, und sicher hat ihn dieser Schritt vor physischem Untergang bewahrt; aristokratisch-preußisch gesonnene Militärs deckten ihn, als er beispielsweise 1936 vom Schwarzen Korps und vom Völkischen Beobachter als «Selbsterreger» angegriffen wurde ${ }^{44}$; daran, daß es in der Wehrmacht solche Strömungen gab, sei mit einem Verweis auf den Widerstand um Stauffenburg erinnert. Benns vorgesetzter General reagierte auf die Attacke vom Schwarzen Korps folgendermaßen: «Er sagte, lassen Sie mir diese Zeitung da, geben Sie mir einige Ihrer Bücher und andere Kritiken über Sie und melden Sie sich in achtundvierzig Stunden bei mir zur Entgegennahme meiner Entscheidung. Diese Entscheidung lautete: Das 'Schwarze Korps' ist ein solches Saublatt, es kann einen offizier gar nicht beleidigen wenn es Sie lobte, wäre es anders - der Fall ist erledigt. Sie bleiben.» ${ }^{45}$

1938 wird Benn von der Reichsschrifttumskammer ausgeschlossen, erhält Schreibverbot mit der Begründung, er verfüge nicht über «die für die Ausübung der schriftstellerischen Tätigkeit erforderliche Eignung» ${ }^{46}$.

Benns Essayistik, seine Briefe, nicht zuletzt seine Gedichte zwischen 1935 und 1945 sind beredtes Zeugnis dafür, daß er die Nazis und die Hitlerei verachtete, und es war auch nicht ganz ungefährlich, was er da in seine Schublade hineinschrieb; für weit Geringeres drohte Konzentrationslager, wenn es nur in falsche Hände geriet. Auch über das Publikationsverbot setzte Benn sich hinweg. 1943 ließ er auf eigene Kosten ein Heft mit 22 Gedichten drucken, das er an Freunde verschickte ${ }^{47}$. Damit soll nicht unterstellt werden, der Dichter Gottfried Benn sei zum Widerstandskämpfer mutiert, er blieb lediglich einer Wahrheit verpflichtet, die, weil sie jenseits des Zeitgeists liegt, nie das ist, was manipulierte Massen für die Wahrheit halten. Wenige tragen das Leid aller; dergleichen sucht sich gewiß niemand aus. «Es gibt fast unerträglich viel, das wir nicht wissen, und der Gründe zum Klagen und Verzweifeln sind Legion. Aber es wird so sein müssen, und es wird unsere Aufgabe bleiben, die Stunde dieser geistigen Welt, solange sie dauert, weiter mit unseren menschlichen Bildern zu erfüllen, so trauerüberladen, so untergangssicher, so monologisch oder so hybrid sie sind.» ${ }^{48}$ - Dies steht auf der letzten Seite von Doppelleben, klingt verhalten optimistisch, ein wenig auch nach Vermächtnis und wird als solches wiederholt in Gottfried Benns letzter autobiographischer Notiz 7956: «Sich absondern - das klingt vielen nicht gut, das klingt vielen provokatorisch und wahlurnenfeindlich, ist es aber nicht, es handelt sich vielmehr um etwas Innerliches, das man lange vergaß: Im Anfang war das Wort, und am Ende wird nicht die Propaganda sein, sondern wieder das Wort.» ${ }^{49}$ 


\section{NOTES}

1. - Cf. K. Pinthus (Hg.), Menschheitsdämmerung, Leipzig 1986, p. 362.

2. - B. Croce, Die Dichtung, Tübingen 1970, p. 118. Die italienische Erstausgabe von La Poesia erschien 1936.

3. - Cf. G. Benn, Gesammelte Werke 8, Wiesbaden 1968, p. 1931, (fortan GW), auch H. Ridley, Gottfried Benn, Opalden 1990, pp. 173-180.

4. - Dichtung ist laut Croce selbstvergessen, hingegeben an das Universale; Literatur hat das Ziel klar im Auge und vergißt niemals die Menschen, an die sie das Wort richtet, und die freilich dem Dichter egal sind. Dichtung werde vom Genius hervorgebracht, der Ingenius (das Talent) produziere Literatur. Cf. B. Croce, Die Dichtung, op. cit, p. 32.

5. - 1921 schreibt Benn Epilog, 1927 Lyrisches Ich, 1928 faßt er beides zu einem Essay zusammen.

Cf. GW 8, p. 2234.

6. - Ibidem, p. 1877. Siebenunddreißig ist Benn 1923/24.

7. - Ibidem 3, p. 569.

8. - F. Nietzsche, Kritische Studienausgabe Bd. 6, München, Berlin, New York 21988, p. 133. (Fortan KSA.)

9. - «Verworrensein und nicht schreiben können, ist noch kein Surrealismus», bemerkt Benn in Doppelleben. GW 8, p. 2020.

10. - «Im Grunde ist jedermann zufrieden, wenn ein Tag vorüber ist. Ihn so ernst zu nehmen, dass er am anderen Tag bereits historische Untersuchungen anstellt, ist lächerlich. Denn damit ist die Hauptlehre, die jeder Tag giebt, verwirkt, 'das Leben ist abzu l e i d e n'». KSA 7, p. 641.

11. - «Ich persönlich bin gegen den Amerikanismus», bekennt Benn 1928. GW 7, p. 1658.

12. - P. Sloterdijk, Kritik der zynischen Vernunft, Zweiter Band, Frankfurt / Main 1983, pp. 741/742.

13. - Laut Alice Miller sind die Deutschen durch ihre autoritäre Erziehung zur Gewalt prädisponiert. Hitler erschien ihnen deshalb als eine Art Messias, weil er die Gewalt auf die anderen, die Juden lenkte. Cf. A. Miller, Am Anfang war Erziehung, Frankfurt/Main 1983, pp. 82-112 sowie pp. 161-231. Dies korrespondiert mit Horkheimer/Adorno, die Antisemitismus als falsche Projektion beschreiben. Cf. M. Horkheimer/Th. W. Adorno, Dialektik der Aufklärung, Leipzig 1989, pp. 209-223.

14. - Aus dem Gedicht Eure Etüden (1954), in: G. Benn, Gedichte, In der Fassung der Erstdrucke, Frankfurt/Main 1982, p. 455.

15. - «Der Gegensatz zu Spiel ist nicht Ernst, sondern - Wirklichkeit.» S. Freud, Der Dichter und das Phantasieren, in: S. Freud, Studienausgabe, Band X, Frankfurt/Main 1982, p. 171.

16. - Cf. GW 8, p. 1882.

17. - Lyrisches Pendant dazu ist das Gedicht Turin (1936). Cf. G. Benn, Gedichte, op. cit., p. 271.

18. - GW 8, p. 1883.

19. - Ibidem, p. 1884.

20. - Zur Funktion der Massenmedien cf. P. Sloterdijk, Krtik der zynischen Vernunft, Zweiter Band, op. cit., pp. 570-575, 893-897.

21. - «Denn seit es bürgerliche Kunst gibt derart, daß die Künstler ohne Protektoren ihr Leben erwerben müssen, haben sie neben der Autonomie ihres Formgesetzes insgeheim das Marktgesetz anerkannt und für Abnehmer produziert.» Th. W. Adorno, Noten zur Literatur I, Frankfurt/Main 1973, p. 150.

22. - Zitiert bei: Peter Schünemann, «Im Dunkel leben, im Dunkel tun was wir können», in: Text + Kritik 44 (1974), p. 10.

23. - GW 8, p. 1936. 
24. - G. Benn, Briefwechsel mit Paul Hindemith, Frankfurt/Main 1986, S. 70. Die Hervorhebungen sind von Benn. «Amor fati» ist eines seiner Lieblingszitate, kehrt in poetisch abgewandelter Form beispielsweise wieder im Gedicht Nur zwei Dinge:

«es gibt nur eines: ertrage - ob Sinn, ob Sucht, ob Sage -

dein fernbestimmtes: Du mußt.» G. Benn, Gedichte, op. cit., p. 427. Das Zitat stammt vom späten Nietzsche. KSA 6, p. 436.

25. - Cf. H. Ridley, G. B., op. cit., pp. 14/15.

26. - Cf. u. a. Können Dichter die Welt ändern?, in GW 7, pp. 1669-1678.

27. - Ibidem, p. 1849.

28. - Cf. B. Hillebrand (Hg.), Über Gottfried Benn, Frankfurt/Main 1987, pp. 129-133.

29. - Goebbels, Reden Bd. 1, Düsseldorf 1971, p. 108.

30. - Die utilitaristische Komponente der Philosophie Piatons ist eines der zentralen Probleme der abendländischen Philosophie. Die verheerenden Folgen, die Piatons Sieg über Diogenes von Sinope und die Kyniker heraufbeschworen hat, prägen das gesamte abendländische Denken überhaupt. Cf. dazu: P. Sloterdijk, Kritik der zynischen Vernunft, op. cit. sowie M. Onfray, Cynismes, Paris 1990 sowie C.-W. Weber, Diogenes, Frankfurt/Main, Berlin 1990.

31. - GW 8, p. 1917.

32. - «... - the rest is silence.» Hamlet, V, 2. «Das allein sind meine Leser, meine rechten Leser, meine vorherbestimmten Leser: was liegt am R e $s$ t? - Der Rest ist bloss die Menschheit.» Nietzsche, KSA 6, pp. 167/168.

33. - Cf. E.R. Curtius, Kritische Essays zur europäischen Literatur, Frankfurt / Main 1984, p. 157.

34. - Der «Fall» Christa Wolf und der «Fall» Heiner Müller seien stellvertretend als jüngste Beispiele genannt. Das hinderte freilich die BILD-Zeitung nicht daran, Heiner Müller nach dessen Ableben in einer Artikelserie auf der VIP-Seite als Genie zu feiern, was nicht verwunderlich ist, denn mittlerweile gibt es kein Medium in Deutschland, das Subversiveres über die Deutschen aussagt als BILD. Hier hat Zynismus sich soweit verselbständigt, daß er der Wahrheit näher kommt als intellektoider Enthüllungsjournalismus, der beständig den am Boden liegenden Hektor schleift. Ob in bezug auf BILD mit Marx zu sagen wäre: «Sie wissen das nicht, aber sie tun es» (MEW 23, p. 88), sei durchaus dahingestellt.

35. - G. Benn, Ausgewählte Briefe, Frankfurt/Main 1986, p. 135.

36. - J. Schröder, Gottfried Benn und die Deutschen, Tübingen 1986, p. 29.

37. - Zu Curtius cf. P. Jehle, Die Politik der Philologen, in : Grenzgänge 4 (1995), pp. 105-128.

38. - E. Buddeberg, Gottfried Benn, Stuttgart 1961, p. V.

39. - Ibidem, p. 93.

40. - Cf. ibidem.

41. - GW 8, p. 1940.

42. - Cf. ibidem, p. 1954.

43. - Cf. ibidem, p. 1960.

44. - Cf. Der Selbsterreger!, in : Über Gottfried Benn, op. cit., pp. 136-140.

45. - GW 8, p. 1963.

46. - Cf. ibidem, p. 1969.

47. - Cf. ibidem, p. 1975.

48. - Ibidem, p. 2038.

49. - Ibidem, p. 2042. 


\section{RÉSUMÉS}

Seit Ende der Zwanzigerjahre war Gottfried Benn Angriffen politischer Eiferer ausgesetzt, gegen die er sich zu verteidigen hatte. Der Dichter selbst, über dessen herausragenden Rang innerhalb der deutschen Dichtung unseres Jahrhunderts kein Zweifel besteht, sah sich nie als politischen Menschen. Mit der Herrschaft des Nationalsozialismus wird Benns Lage existentiell bedrohlich. Er unterschätzt kurzzeitig die kriminelle Energie der neuen Machthaber und glaubt, von ihnen in Ruhe gelassen zu werden, bevor er mit dem Eintritt in die Armee als Arzt die «aristokratische Form der Emigration» wählt.

Infolge seines kurzen Irrtums im Jahre 1933 wurde nach der Nazizeit Benn häufig als Vehikel mißbraucht, um die in Deutschland nie bewältigte Nazi Vergangenheit in den Griff zu bekommen.

Gottfried Benns autobiographische Schriften, die ihm sämtlich mehr oder weniger abgenötigt wurden, werden im politischen Spannungsfeld, dem Benn zeitlebens ausgesetzt war, als Versuch des Dichters gesehen, der kollektiven germanischen Paranoia durch Wahrheit entgegenzuwirken.

Attaqué par les fanatiques politiques depuis la fin des années vingt, Gottfried Benn fut obligé de se défendre. Lui même ne se considéra jamais comme quelqu'un de politisé. Dès la prise du pouvoir par les nazis, Benn se trouva rapidement exposé à divers dangers dont certains menaçaient son existence même. Il sous-estima, pendant un moment très bref, l'énergie criminelle dont était capable un nouveau régime politique dont il espérait qu'il ne l'inquiéterait pas outre-mesure. Puis il finit par choisir « la forme aristocrate de l'exil » en s'engageant comme médecin dans la Wehrmacht.

Du fait de son erreur en 1933, Benn fit longtemps figure de bouc émissaire susceptible d'aider les Allemands à surmonter un passé nazi sur lequel la société allemande n'avait jamais véritablement accompli de travail du deuil.

Les textes autobiographiques que Benn s'est vu contraint d'écrire sont analysés ici en tant que tentative d'un écrivain, pris toute sa vie dans les tensions du champ politique, de faire pièce à la paranoïa germanique collective en recourant à la vérité.

\section{AUTEUR}

\section{RAMMON REIMANN}

Université Charles-de-Gaulle - Lille III 